\title{
A Method for Inversion of Smooth Open Boundary Conditions
}

\author{
Haidong Pan, Zheng Guo and Xianqing Lv ${ }^{*}$ \\ Physical Oceanography Laboratory, Ocean University of China, Qingdao 266003, China \\ ${ }^{*}$ Corresponding author
}

\begin{abstract}
A new method is developed as a solution for ill-posedness problem in inversion of open boundary conditions (OBCs). In this method, open boundary curves are parameterized by interpolating values at some selected independent points(IPs) with the Spline. Compared with the linear interpolation traditionally used in OBCs inversion, curves obtained with the new method is more smooth and more consistent with the ocean reality. The model and the method are evaluated in twin experiment(TE).In practical experiment(PE), the $T / P$ satellite observations are assimilated to simulate tides in the Bohai and Yellow Seas(BYS). Mean absolute errors (MAEs) of practical experiment with the new method are smaller than those with the traditional.
\end{abstract}

Keywords-adjoint method; open boundary conditions; independent points; spline

\section{INTRODUCTION}

Appropriate $\mathrm{OBCs}$ are required for tides and tidal currents simulation by regional ocean models. Traditionally, origins of OBCs fall into two categories: interpolation of observations near the open boundary obtained from tidal stations and satellites; simulation results of larger scale models. However, determination of OBCs remains as a great challenge due to scarceness of available observations and low resolution or accuracy of larger scale models. After the adjoint assimilation method was introduced into oceanography, many researchers explored feasibility and performance of the method in obtaining OBCs [1-6]. With the adjoint method, OBCs can be adjusted such that the simulation results come close to the available observations.

In some previous works on inversion of OBCs, values at all grid points along the open boundary are regarded as control variables, which may result in inaccurate $\mathrm{OBC}$ due to the ill-posedness of the inverse problem[7]. Cao et al. [8] and Chen et al. [9] put forward the Independent Points Strategy as a solution. They take values at a set of selected grid points as control variables and those at other points can be calculated through linear interpolation. Zhang and Wang[10] improve the strategy by selecting feature points as IPs, which reduces the number of control points to the minimum. However, open boundary curves obtained with linear interpolation in these works are not smooth, which is different from the reality. In this study, a new method has been developed as an improvement on the IPs Strategy: values at IPs are interpolated using the cubic Spline. As the variations of tidal harmonic constants are generally smooth, open boundary curves obtained with the Spline interpolation can be more close to the real one.

This paper is organized as follows. Optimization of IPs along the open boundary with the new method is presented in Section 2. The twin experiments with a rectangular computing area are described in Section 3. In Section 4, practical experiments are carried out to further test performance of the new method. Finally, conclusions are given in Section 5.

\section{METHODOLOGY}

The two-dimensional adjoint tidal model in Lu and Zhang [11] is used in this study. Under the assumption that the OBC is fully spatially varying, which means all grid points along the OBC are taken as IPs, the correction of Fourier coefficients at IPs is as follows[8]:

$$
\begin{aligned}
& K\left(a_{i, l}-a_{i, l}^{\prime}\right)+\sum_{j=1}^{J} T_{l}^{j} \cos \left(\omega_{l} j \Delta t\right)=0 \\
& K\left(b_{i, l}-b_{i, l}^{\prime}\right)+\sum_{j=1}^{J} T_{l}^{j} \sin \left(\omega_{l} j \Delta t\right)=0
\end{aligned}
$$

where $\omega_{i}$ is the frequency of the $i$-th tidal constituent. $a_{i, l}$ and $b_{i, l}$ are Fourier coefficients at the $l$-th IP.

Fourier coefficients can be calculated for each tidal constituent following the same procedure. For clarity, we neglect the subscript of constituent number $i$ in the following equations. Interpolate $a_{l}$ and $b_{l} \quad(l=1, \ldots, \mathrm{L}$, where $\mathrm{L}$ denotes IPs number), Fourier coefficients at IPs, with the Spline interpolation, we can obtain values at the $k$-th point along the open boundary.

Eventually, the correction of Fourier coefficients at IPs in this study can be obtained:

$$
\begin{aligned}
& K\left(a_{l}-a_{l}^{\prime}\right)+\sum_{l=1}^{L} f_{k, l}\left(\sum_{j=1}^{J} T_{k}^{j} \cos (\omega j \Delta t)\right)=0 \\
& K\left(b_{l}-b_{l}^{\prime}\right)+\sum_{l=1}^{L} f_{k, l}\left(\sum_{j=1}^{J} T_{k}^{j} \sin (\omega j \Delta t)\right)=0
\end{aligned}
$$

where $f_{k, l}$ is the weighting coefficients calculated by Spline method. IPs on OBCs can be updated by (3) and (4). 


\section{TWIN EXPERIMENTS}

\section{A. Setup of TE}

In this section, twin experiments are conducted to test the feasibility and performance of the method developed. An ideal 40-meter-deep rectangular ocean $\left(34^{\circ}-41^{\circ} \mathrm{N}\right.$, $117^{\circ} 40^{\prime}-123^{\circ} 30^{\prime} \mathrm{E}$ ) with southern open boundary is designed for these experiments. The model uses the Arakawa C-Grid with a horizontal resolution of $10^{\prime} \times 10^{\prime}$. The Coriolis parameter $f$ takes the local value, bottom friction is set to be 0.002 and the time step interval to be 372.618 s, $1 / 120$ of a M2 tidal cycle. Because the study focus on development of the method, only the M2 tide is calculated.

Experimental procedure is as follows. Run the forward model with prescribed OBCs and record the simulated results at 64 selected grid points as 'observations'. Assign initial values to Fourier coefficients at IPs to run the forward model, and then the differences between simulated results and observations can be calculated, serving as external force for the adjoint model. After backward integration of the adjoint equations, OBCs can be updated by (3) and (4). Repeat these steps and OBCs can be continuously optimized until certain criterion is met.

To compare effects of linear and Spline interpolation on inversion, Cressman and Spline methods are adopted when three uniformly distributed IPs are used. Two prescribed distributions of Fourier coefficient $b$ along the open boundary are shown in Figure 1 while a is set to be constant as 0 , which will be inverted in experiments TE1-1 and TE1-2 respectively.

\section{B. Result and Discussion of TE}

The values of cost function normalized by those at the first iteration step serve as an important criterion to assess the inversion. Decreasing process of the cost function in TE1 are shown in Figure 2. Compared with the Cressman method, the Spline interpolation results in rapid decrease of the cost function.
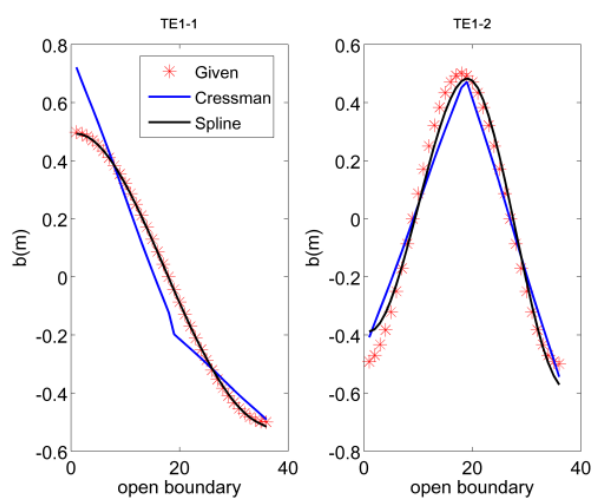

FIGURE I. PRESCRIBED OBCS AND INVERTED OBCS

Inverted OBCs (Figure 1) show that the curve obtained with the Spline interpolation is more smooth than with the linear interpolation. The misfits between the inverted value and the prescribed one averaged along the open boundary are given in Table 1. It can be concluded that the new method is better than the traditional one when the same number of IPs are used.

The MAE of amplitude, phase and magnitude of vector difference(MVD) in TE1 are presented in Table 2. MAE of twin experiments with the new method are smaller than those with the traditional, which further confirms the conclusion drawn from Table 1.

TABLE I. THE MISFIT BETWEEN THE INVERTED AND PRESCRIBED OBC IN TE1 (UNITS: CM)

\begin{tabular}{ccc}
\hline $\begin{array}{c}\text { Interpolation } \\
\text { method }\end{array}$ & TE1-1 & TE1-2 \\
\hline Cressman & 7.2 & 6.7 \\
Spline & 0.3 & 4.2 \\
\hline
\end{tabular}

TABLE III. THE MAE OF THE OBSERVATIONS IN THE PRACTICAL EXPERIMENT

\begin{tabular}{cccc}
\hline $\begin{array}{c}\text { Interpolation } \\
\text { method }\end{array}$ & $\begin{array}{c}\text { Amplitude } \\
(\mathrm{cm})\end{array}$ & $\begin{array}{c}\text { Phase } \\
\left({ }^{\circ}\right)\end{array}$ & $\begin{array}{c}\text { MVD } \\
(\mathrm{cm})\end{array}$ \\
\hline Cressman & 4.2 & 3.4 & 7.1 \\
Spline & 3.5 & 3.2 & 6.1 \\
\hline
\end{tabular}

TABLE II. MAE OF AMPLITUDE, PHASE AND MAGNITUDE OF VECTOR DIFFERENCE

\begin{tabular}{ccccccc}
\hline $\begin{array}{c}\text { Interpolation } \\
\text { method }\end{array}$ & TE1-1 & \multicolumn{3}{c}{ TE1-2 } \\
\cline { 2 - 7 } & $\begin{array}{c}\text { Amplitude } \\
(\mathrm{cm})\end{array}$ & $\begin{array}{c}\text { Phase } \\
\left({ }^{\circ}\right)\end{array}$ & $\begin{array}{c}\text { Magnitude of } \\
\text { vector } \\
\text { difference }(\mathrm{cm})\end{array}$ & $\begin{array}{c}\text { Amplitude } \\
(\mathrm{cm})\end{array}$ & $\begin{array}{c}\text { Phase } \\
\left({ }^{\circ}\right)\end{array}$ & $\begin{array}{c}\text { Magnitude of } \\
\text { vector } \\
\text { difference }(\mathrm{cm})\end{array}$ \\
\hline Cressman & 1.7 & 5.5 & 2.38 & 1.0 & 3.1 & 1.71 \\
Spline & 0.1 & 0.2 & 0.11 & 0.5 & 1.6 & 0.97 \\
\hline
\end{tabular}




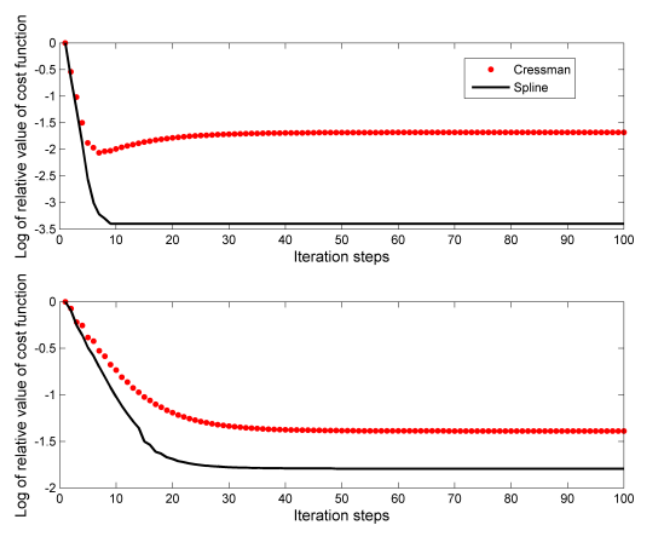

FIGURE II. DECREASING PROCESS OF COST FUNCTION IN TE1.

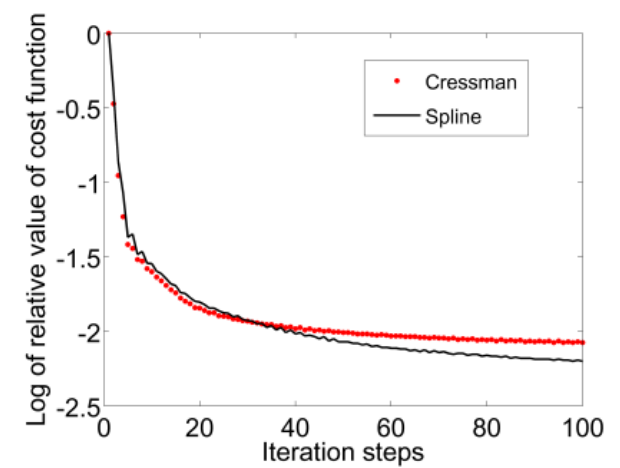

FIGURE III. DECREASING PROCESS OF COST FUNCTION IN PRACTICAL EXPERIMENTS.
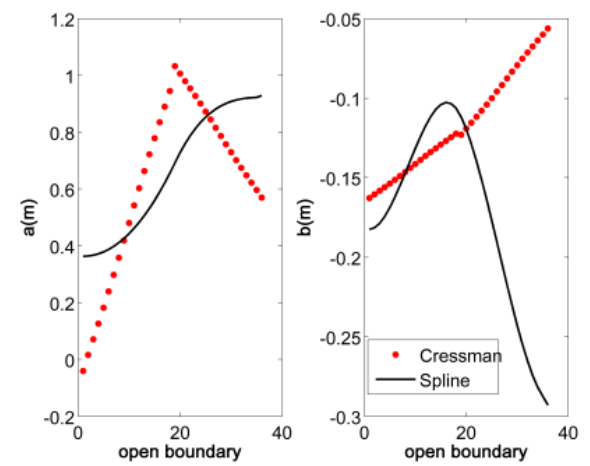

FIGURE IV. OPTIMIZED OBCS OF PE

\section{PRACTICAL EXPERIMENTS}

The setup of practical experiment (PE) is the same with TE except that the bathymetry of BYS and positions of real observations along the T/P track are adopted. In this section, harmonic constants obtained from $\mathrm{T} / \mathrm{P}$ altimetry are assimilated into the model to assess the method in practical application. The IP strategies are the same with those in TE.

Figure 3 shows the decreasing process of normalized cost function. After 100 iteration steps, the cost function reduces to 1 percent of its initial value for both interpolation methods. Inverted $\mathrm{OBCs}$ are given in Figure 4, varying with interpolation methods. Table 3 shows the MAE between simulated values and observations. The MAE of amplitudes, phases, and magnitude of difference vector with Spline method are smaller than those with Cressman interpolation. Co-tidal charts based on model results obtained with two interpolation methods are given in Figure 5. As can be seen from both cocharts, there are four amphidromic points in the computation region, which are located near Qinhuangdao, Yellow River delta, Chengshantou and Jiangsu.
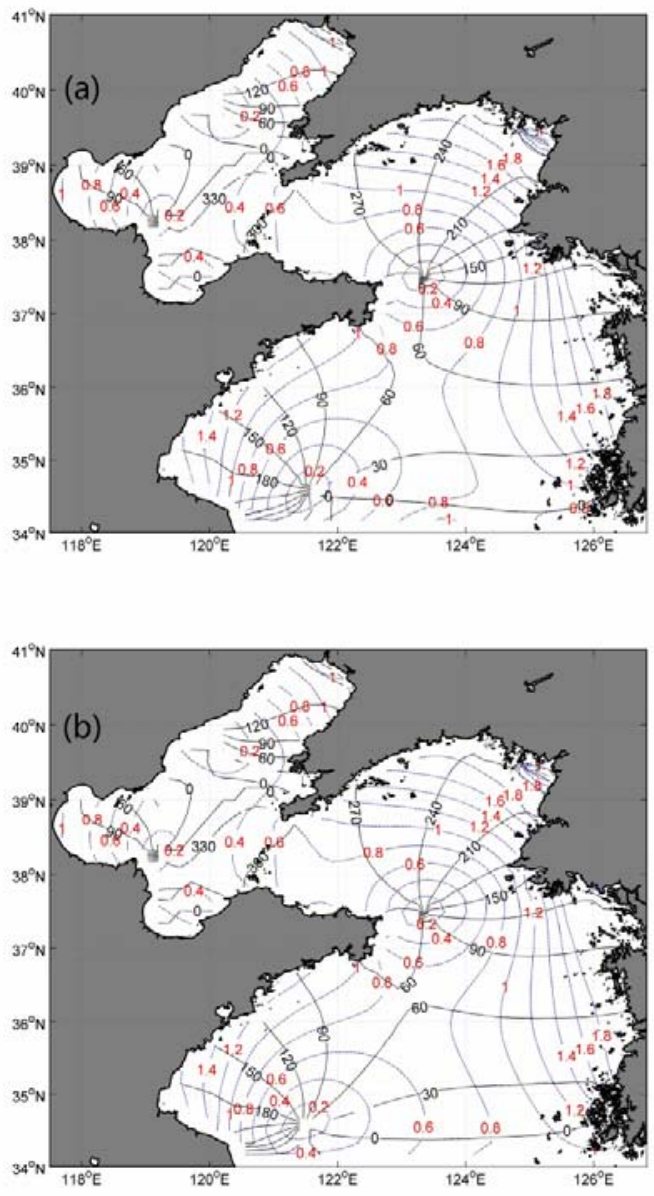

\section{FIGURE V. CO-TIDAL CHARTS FOR M2 CONSTITUENT IN THE} BYS.(a) CRESSMAN (b) SPLINE

\section{CONCLUSIONS}

Determination of OBCs in the regional ocean model has been an on-going research subject. OBCs obtained from available observations or simulated results of the larger ocean model often requires manual adjustment, which is heavily dependent on researchers' experience. The adjoint method has been widely applied in oceanography as a powerful tool for parameter estimation. In some studies on OBCs inversion, all grid points along the open boundary are taken as IPs, which results in the ill-posedness problem. The IPs strategy serves as an effective solution for this problem.

As an improvement on IPs strategy, a new method has been proposed in this study, in which open boundary curves are obtained by interpolating values at IPs with the cubic 
spline instead of linear method. To test feasibility of the new method, twin experiments are carried out. The results show that, with the new method, inverted curves along the open boundary are more consistent with the prescribed ones and the misfit between simulated results and observations is smaller. In practical experiments, M2 tidal constituent in the BYS is simulated with assimilation of $\mathrm{T} / \mathrm{P}$ satellite data. Co-tidal chart based on simulated results show M2 constituent characteristics, which is consistent with previous studies.

\section{ACKNOWLEDGMENTS}

The authors would like to acknowledge the funding support of this research by the Natural Science Foundation of Shandong Province of China through Grant ZR2014DM017, the National Natural Science Foundation of China through grant 41606006 and 41371496, the Natural Science Foundation of Zhejiang Province through Grant LY15D060001, the State Ministry of Science and Technology of China through grant 2013AA09A502, the National Science and Technology Support Program through grant 2013BAK05B04, and the Ministry of Education's 111 Project through Grant B07036.

\section{REFERENCES}

[1] R. W. Lardner, A. H. Al-Rabeh, and N. Gunay, "Optimal estimation of parameters for a two-dimensional hydrodynamical model of the Arabian Gulf,’J. Geophys.Res.,vol.98,no.10,pp.18229-18242, 1993.

[2] U. Seiler, "Estimation of open boundary conditions with the adjoint method," J. Geophys.Res., vol. 98, no. 12, pp. 22855-22870, 1993.

[3] A.W.Heemink,E.E.A.Mouthaan,M.R.T.Roest,E.A.H.Vollebregt, K.B. Robaczewska, and M. Verlaam, "Inverse 3D shallow water flow modeling of the continental shelf," Cont. Shelf Res., vol.22,no.3,pp.465-484, 2002.

[4] A. Zhang, B. B. Parker, and E. Wei, "Assimilation of water level data into a coastal hydrodynamic model by an adjoint optimal technique," Cont.Shelf Res., vol. 22, no. 14, pp. 1909-1934, 2002.

[5] N. Ayoub, "Estimation of boundary values in a North Atlantic circulation model using an adjoint method,"Ocean Model.,vol.12,no.3-4,pp.319-347, 2006.

[6] J.C. Zhang, and X.Q. Lu, "Parameter estimation for a three-dimensional numerical barotropic tidal model with adjoint method," Int. J. Numer. Methods. Fluids ,vol.57 ,no.1,pp.47-92, 2008.

[7] L. S. Yu, and J. J. O'Brien, "Variational estimation of the wind stress drag coefficient and the oceanic eddy viscosity profile," J. Phys. Oceanogr. , vol.21, no.5, pp.709-719, 1991

[8] A.Z.CAO, Z.Guo, and X.Q.Lu, "Inversion of two-dimensional tidal open boundary conditions of M2 constituent in the Bohai and Yellow Seas," Chin.J. Oceanol. Limn.,vol.30, no.5,pp.868-875, 2012.

[9] H. B. Chen ,A. Z. Cao, J. C. Zhang, C. B. Miao, and X. Q. Lu, "Estimation of spatially varying open boundary conditions for a numerical internal tidal model with adjoint method," Math. Comput. Simul. vol.97,pp.14-38, 2014.

[10] J. C. Zhang, and Y. P. Wang, "A method for inversion of periodic open boundary conditions in two-dimensional tidal models," Comput.Method. Appl. M, vol.275, pp.20-38, 2014.

[11] X.Q.Lu, and J.C. Zhang, "Numerical study on spatially varying bottom friction coefficient of a 2D tidal model with adjoint method," Cont. Shelf Res. ,vol.26 ,no.16,pp.1905-1923, 2006. 